\title{
Impacto en el patrón de atención en cirugía de cabeza y cuello y las medidas sanitarias adoptadas durante los primeros 150 días de la era COVID-19
}

\author{
Karina Toledo-Villegas ${ }^{1}$, Angelica Silva-Figueroa ${ }^{1}$, Rodrigo Castro S. ${ }^{1}$, Oscar Aguilar C. ${ }^{1}$, \\ Rene Inturias C. ${ }^{1}$, Alejandra Gallego C. ${ }^{1}$ y Miguel González-Prado ${ }^{1}$
}

Servicio y Departamento de Cirugía, Hospital Barros LucoTrudeau. Santiago, Chile.

Este estudio fue aprobado por el comité ético-científico del Servicio de Salud

Metropolitano Sur (SMSS)

y Dirección del Complejo Asistencial Barros Luco (CABL)

Recepción 2021-01-23, aceptado 2021-03-25

Correspondencia a: Dra. Angelica Silva-Figueroa angelica.silva@uft.c
Impact on the pattern of care in Head and Neck Surgery and the regional and institutional policy changes adopted during the first 150 days of the COVID-19 era

Introduction: The COVID-19 pandemic generated a restructuring of surgical care worldwide due to the disease's high transmissibility and the inherent limitation of available human and material resources. Aim: The study's aim was to describe the impact of the COVID-19 pandemic on the head and neck surgery team at Complejo Asistencial Barros Luco Trudeau (CABL) in clinical-surgical execution and organization of sanitary sequencing measures implemented over time during the first 150 days after the pandemic started in Chile. Materials and Method: We performed a retrospective review of patients undergoing surgery or outpatient evaluation during the COVID-19 period from 03-03-2020 to 07-31-2020, compared to the same time interval in 2019. Clinical characteristics and sanitary measures used during this period were synthesized. Results: We detected a $64 \%$ decrease in outpatient care and a 58\% decrease in surgical load from 2019. During the COVID-19 period of 2020, a total of 61 patients underwent surgical intervention. The main indication for surgery was cancer, in $75.4 \%$ of patients (46). COVID-19 was not reported in any patients in the 14 days following hospitalization. We discussed the perioperative considerations used and the national/ institutional sanitary restrictions. Conclusions: The global health crisis to COVID-19 generated a reduction in outpatient care and surgeries performed by the CABL head and neck team. Despite health restrictions, we organized care stratified to preserve critical head and neck non-deferrable cases.

Key words: COVID-19; SARS-CoV-2; head and neck surgery; oncology.

\section{Resumen}

Introducción: La pandemia COVID-19 generó una reestructuración en la atención quirúrgica mundialmente debido a su alta transmisibilidad y la inherente limitación de los recursos humanos y materiales disponibles. Objetivo: Describir el impacto de la pandemia COVID-19 en el Equipo de Cirugía Cabeza y Cuello del Complejo Asistencial Barros Luco Trudeau (CABL) en su ejecución clínico-quirúrgica y la secuenciación organizada de las medidas sanitarias aplicadas a lo largo del tiempo durante los primeros 150 días de iniciada la pandemia en Chile. Materiales y Método: Realizamos una revisión retrospectiva de los pacientes sometidos a cirugía y/o evaluados ambulatoriamente durante el período COVID-19 comprendido entre el 3 de marzo y el 31 de julio de 2020, comparado con el mismo intervalo de tiempo de 2019. Características clínicas y medidas sanitarias empleadas durante este período fueron sintetizadas. Resultados: Detectamos un descenso del 64\% en atención ambulatoria y un descenso del 58\% en la carga quirúrgica, comparado con el año 2019. Durante el período COVID-19 de 2020, un total de 61 pacientes fueron sometidos a intervención quirúrgica. La principal indicación de cirugía fue cáncer en un 75,4\% (46). No se reportaron pacientes contagiados por COVID-19 en los 14 días siguientes a la hospitalización. Se discuten las consideraciones perioperatorias empleadas y restricciones nacionales/institucionales sanitarias. Conclusión: La crisis sanitaria mundial secundaria al COVID-19 generó una reducción en las atenciones ambulatorias y cirugías realizadas por Equipo de Cabeza y Cuello CABL. A pesar de las restricciones sanitarias, organizamos estratificadamente la atención para preservar la resolución de casos críticos no diferibles en cabeza y cuello.

Palabras clave: COVID-19; SARS-CoV-2; cirugía de cabeza y cuello; oncología. 


\section{Introducción}

Desde iniciada la pandemia COVID-19, hospitales y cirujanos fueron advertidos de posponer cirugías electivas hasta la disminución de la curva de contagios, de manera tal que la infraestructura y recursos humanos de los centros de salud pudiesen cubrir las necesidades de los pacientes afectados por la pandemia, que se disemina rápidamente ${ }^{1,2}$. Algunos de los más grandes desafíos que se deben solventar son: la disminución de camas hospitalarias disponibles, la redistribución del recurso humano y el limitado equipamiento de protección personal (EPP), sin contar las altas tasas de mortalidad posoperatorias reportadas en cirugías electivas de pacientes en la fase prodrómica de la infección por SARS-CoV-2, con una incidencia extremadamente alta de hospitalización en UCI del 44\%-51\% y una mortalidad del 20\%-38\% $\%^{3-6}$. Además, los pacientes oncológicos no estuvieron excluidos de estos resultados negativos ante procedimientos quirúrgicos cercanos a la adquisición de infección por SARS-CoV-2, y sin considerar que en algunos casos el retraso en sus tratamientos pudiera ser también deletéreo oncológicamente en cirugía de cabeza y cuello ${ }^{7-9}$.

A causa de las razones expuestas previamente y para poder mantener la actividad quirúrgica, aminorando los riesgos de transmisibilidad y comorbilidad quirúrgica del COVID-19, es que distintas sociedades de cirugía de cabeza y cuello a nivel mundial han redactado guías de recomendación para la toma de decisiones en la elección de pacientes y protección del personal en distintos escenarios clínicos ${ }^{8-18}$. Hasta el momento no existen publicaciones nacionales que informen de la repercusión del período COVID-19 en cirugía de cabeza y cuello en nuestro medio durante este período, ni las medidas sanitarias adoptadas para aminorar los riesgos durante la pandemia. Por lo tanto, el objetivo de la presente publicación es describir el impacto de la pandemia COVID-19 en el Equipo de Cirugía de Cabeza y Cuello (ECCC) del Complejo Asistencial Barros Luco Trudeau (CABL) en la ejecución de cirugía de cabeza y cuello, atención ambulatoria, y la secuenciación organizada de las medidas sanitarias aplicadas a lo largo del tiempo para el correcto desempeño de los procedimientos quirúrgicos en Cirugía de Cabeza y Cuello, aplicados durante los primeros 150 días de iniciada la pandemia en Chile.

\section{Materiales y Método}

\section{Medidas sanitarias Nacionales y Locales CABL}

Se recopilaron las directrices sanitarias nacionales de la Subsecretaría de Redes Asistenciales y
Gobierno de Chile ${ }^{19-23}$ desde el primer caso reportado en Chile hasta el 30 de julio de 2020, y las medidas secuenciales empleadas por CABL y del ECCC para mantener la atención de los usuarios.

\section{Pacientes}

Se realizó revisión retrospectiva de fichas clínicas de todos los pacientes intervenidos por el ECCC en el CABL en el período comprendido entre el 3 de marzo y el 31 de julio de 2020, considerándose fecha de inicio del estudio el primer caso de COVID-19 confirmado en Chile. Los criterios de exclusión fueron información médica incompleta.

\section{Atención abierta y cerrada}

Se recolectaron los datos absolutos de atención abierta y quirúrgica del período comprendido entre el 03-03-2019 y el 31-07-2019 por el ECCC en el CABL, incluyendo: números de casos nuevos, números de controles presenciales de pacientes conocidos y números de intervenciones quirúrgicas totales realizadas por el equipo.

Con respecto a la infección por COVID-19, se consideró como infección intrahospitalaria por COVID-19 a los pacientes que contrajeron la infección durante los 14 días posterior a la cirugía ${ }^{24}$.

En todos los procedimientos quirúrgicos, el equipo de cabeza y cuello interviniente hizo uso de EPP según las recomendaciones sanitarias nacionales e internacionales ${ }^{22,23,25}$. Se aplicaron recomendaciones de categorización internacionales para intervención quirúrgica en cirugía de cabeza y cuello adaptadas a la realidad nacional ${ }^{14}$.

Este estudio fue aprobado por el comité éticocientífico del Servicio de Salud Metropolitano Sur y Dirección del CABL.

\section{Resultados}

\section{A nivel nacional sanitario}

Luego de reportado el primer caso COVID-19 chileno, el Gobierno de Chile emitió la alerta sanitaria nacional el 6 de marzo de 2020. Finalmente, se emitió Plan de Contingencia COVID-19 para operacionalizar la atención categorizada en salud en todos los centros de salud, con las siguientes instrucciones:

- Suspensión de las cirugías electivas.

- Reforzamiento del área de urgencia y redistribución de la atención cerrada hospitalaria en recursos humanos y equipamiento.

- Formación de polichoques según especialidad con prioridad. 


\section{A nivel institucional $C A B L$}

Medidas:

- Las 3 áreas quirúrgicas de CABL: Pabellón Central, Placa Técnica y la Unidad de Cirugía Ambulatoria (UCA), fueron redistribuidas durante la pandemia como zona Libre de COVID-19, área COVID-19 (+)/urgencias y paciente ambulatorio libre de COVID-19 respectivamente.

- Redistribución de camas y recurso humano para la atención de pacientes COVID-19; camas de baja complejidad, camas de alta complejidad (UCI/UTI) y salas de operaciones.

- Comités oncológicos multidisciplinarios CABL, implementaron desde el 16 de marzo de 2020 reuniones semanales vía online para presentación de casos oncológicos.

\section{Consideraciones preoperatorias del Equipo de Cirugía de Cabeza y Cuello CABL}

Para este período COVID-19 se implementaron la Encuesta de Riesgo de Enfermedad por Coronavirus Preoperatoria (ERECoVp), PCR SARS-CoV-2 preoperatoria, y se agregó un addendum al consentimiento informado preoperatorio.

La ERECoVp confeccionada comenzó a aplicarse el 22 de marzo de 2020 en todo paciente planificado para cirugía por el equipo y consistió en un tamizaje activo de riesgo de COVID-19 de los pacientes en su etapa prequirúrgica telefónicamente en ambiente prehospitalario e intrahospitalariamente junto al consentimiento informado por el ECCC (suplemento $\mathrm{n}^{\mathrm{o}} 1$ ). Todos los pacientes fueron sometidos a medición de temperatura e ingresaban en modalidad ambulatoria.

La implementación de PCR SARS-CoV-2 preoperatoria fue operacionalizada en CABL el 12 de mayo de 2020, inicialmente con 2 cupos por día para lunes y jueves, con plazo máximo de realización de PCR SARS-CoV-2 preoperatoria de 48 a 72 horas previo a la cirugía programada. Cada una de estas pruebas se realizarían en el centro respiratorio. Además, se confeccionó un addendum al consentimiento informado considerando los pacientes asintomáticos/PCR SARS-CoV-2 falsos negativos.

Las medidas ministeriales del CABL y del Equipo, más críticas, fueron resumidas cronológicamente en la Tabla 1 y se realizó una representación gráfica con número de casos COVID-19 nuevos por día en Chile y las medidas sanitarias más relevantes en la Figura 1.

Durante el período 2020 de este estudio hubo 3 casos que rechazaron cirugía oncológica y 2 casos se encontraban en cuarentena por ser contacto cercano de un familiar COVID-19 positivo. Hubo un solo caso programado para cirugía que durante su control de signos vitales intrahospitalario se detectó temperatura digital de $38^{\circ} \mathrm{C}$ con encuesta COVID-19 telefónica negativa, y cuyo cuadro febril fue secundario a una infección urinaria febril.

\section{A nivel del área quirúrgica}

El $90 \%$ de las cirugías realizadas durante el período estudiado se realizó en el Pabellón Central CABL, un 6,5\% se realizó en UCA y sólo un 3,5\% en el área de pabellones COVID-19 (+).

Con respecto a los números absolutos de cirugías durante los períodos del 3 de marzo al 30 de julio en 2019 y 2020, respectivamente, son representados en la Tabla 2. Las intervenciones quirúrgicas de 2019 a 2020 se redujeron en un 58\%. En 2019 la tasa promedio de cirugía programada fue del 6,5 (rango $5,0-9,0$ ) cirugías por semana y en 2020 de 2,8 (rango $0,0-6,0)$ cirugías por semana en ECCC del CABL. En 2020 el $75 \%$ de las intervenciones quirúrgicas fueron por cáncer.

En 2020, en total se identificaron 61 usuarios que fueron sometidos a intervención quirúrgica durante el período de estudio. Las características demográficas y clínicas de los pacientes están enumeradas en la Tabla 3. El 67,2\% de los pacientes sometidos a cirugía programada fueron sometidos a encuesta de riesgo de COVID-19 intrahospitalaria preoperatoria; de los cuales $12(29,2 \%)$ fueron sometidos a ERECoVp y PCR SARS-CoV-2 preoperatoria simultáneamente, y $29(70,7 \%)$ fueron sometidos exclusivamente a encuesta de riesgo de COVID-19. No se sometieron a ERECoVp ni PCR SARSCoV-2 preoperatoria 20 pacientes $(32,8 \%)$.

No se registró mortalidad asociada a COVID-19 en los pacientes estudiados y la causa de muerte de los 3 pacientes fallecidos de esta serie fueron: liposarcoma de alto grado occipital irresecable con ECOG 4, cáncer pulmonar de células pequeñas y adenocarcinoma poco diferenciado de glándulas salivales con síndrome de vena cava, ECOG 3.

\section{A nivel de área ambulatoria}

El Centro de Diagnóstico y Tratamientos (CDT) CABL implementó en el servicio de atención ambulatoria quirúrgica desde el 18 de marzo de 2020:

- Sistema de categorización (TRIAGE) según patología de consulta/derivación, detección de temperatura y encuesta de síntomas COVID-19.

- Citaciones presenciales para evaluación por especialidad quirúrgica, luego de TRIAGE negativo, eran con mascarilla y sin acompañantes, con excepción a los casos que requerían asistencia personal de terceras personas. 
Tabla 1. Cronograma general de cambios sanitarios críticos ministeriales, institucionales y Equipo de Cirugía de Cabeza y Cuello del Complejo Asistencial Barros Luco (CABL) desde el 03.03.2020 al 30.07.2020

\begin{tabular}{|c|c|c|c|}
\hline Políticas ministeriales en relación a COVID-19 & $\begin{array}{c}\text { Medidas instauradas en } \\
\text { CABL }\end{array}$ & $\begin{array}{l}\text { Medidas instauradas por } \\
\text { Equipo C y C }\end{array}$ & $\begin{array}{l}\text { Número de casos } \\
\text { COVID-19 nuevos } \\
\text { nacional por día* }\end{array}$ \\
\hline \multicolumn{3}{|c|}{ 06.03.2020: Decreto Alerta Sanitaria por COVID-19 Emergencia de Salud Pública de Importancia Internacional (ESPII) } & 06.03.2020: 1 \\
\hline 11.03.2020: OMS declara pandemia por COVID-19 & & & 11.03.2020: 6 \\
\hline $\begin{array}{l}\text { 18.03.2020: CVE 1742691 Declaración Estado de } \\
\text { Excepción Constitucional de Catástrofe por Calamidad } \\
\text { Pública } \\
\text { 18.03.2020: Circular C } 37 \mathrm{~N}^{\mathrm{o}} 01 \text { Protocolo de uso correcto } \\
\text { de equipo de protección personal en pacientes sospechosos } \\
\text { o confirmados de COVID-19 } \\
\text { 18.03.2020: ORD. C } 37 \mathrm{~N}^{\mathrm{o}} 670 \text { Herramientas para evaluar } \\
\text { estado de implementación de medidas de prevención de } \\
\text { IAAS asociados al virus SARS-CoV-2 }\end{array}$ & $\begin{array}{l}\text { 18.03.2020: Instauración } \\
\text { de Triage CDT, } \\
\text { estratificación de atención } \\
\text { ambulatoria presencial } \\
\text { e implementación de } \\
\text { seguimientos telefónicos } \\
\text { en servicio cirugía. } \\
\text { *Los pacientes con } \\
\text { sintomatología de } \\
\text { sospecha eran derivados } \\
\text { al centro respiratorio para } \\
\text { evaluación }\end{array}$ & $\begin{array}{l}\text { 20.03.2020: Plan } \\
\text { de mantención de } \\
\text { atención quirúrgica y } \\
\text { ambulatoria del equipo } \\
\text { según recomendaciones } \\
\text { COVID-19 nacionales } \\
\text { e internacionales } \\
\text { (protocolización) }\end{array}$ & 20.03.2020: 92 \\
\hline $\begin{array}{l}\text { 27.03.2020: ORD. C27/No } 808 \text { Recomendaciones de Plan } \\
\text { de Contingencia COVID-19 } \\
\text { Líneas de acción establecidas por MINSAL: } \\
\text { - No concurrencia de pacientes y familiares a los estable- } \\
\text { cimientos de salud salvo emergencias } \\
\text { - Evitar aglomeración de familiares y personal clínico } \\
\text { - Optimizar los procesos de atención clínico/administrati- } \\
\text { vo para disminuir el tiempo de permanencia de usuarios } \\
\text { en los establecimientos de salud } \\
\text { - Los procesos clínicos diagnósticos y terapéuticos, no } \\
\text { prioritarios ni críticos, deberán postergarse o reprogra- } \\
\text { marse } \\
\text { - Suspender todo tipo de capacitaciones, reuniones, talle- } \\
\text { res y acreditaciones masivas, que no estén en relación } \\
\text { con la contingencia COVID-19 } \\
\text { - Postergación de atención en policlínico } \\
\text { - Implementación de seguimiento telefónico para los pa- } \\
\text { cientes nuevos y controles }\end{array}$ & & $\begin{array}{l}\text { 22.03.2020: Implementa- } \\
\text { ción de encuesta de riesgo } \\
\text { de COVID-19 a pacientes } \\
\text { preoperatorios }\end{array}$ & 22.03.2020: 95 \\
\hline $\begin{array}{l}\text { 30.03.2020: Decreto No } 11 \text { Ministerio de Hacienda: } \\
\text { Suspensión de Garantías Explícitas en Salud de } 67 \\
\text { patologías GES (incluido cáncer de tiroides) con criterios } \\
\text { de excepción }\end{array}$ & $\begin{array}{l}\text { 30.03.2020: Redistribución } \\
\text { de sala de operación } \\
\text { en COVID19 y no } \\
\text { COVID-19, rotaciones de } \\
\text { recurso humano para salas } \\
\text { COVID-19 y conversión } \\
\text { de salas quirúrgicas a } \\
\text { salas COVID-19 }\end{array}$ & $\begin{array}{l}\text { 30.03.2020: Tasa general } \\
\text { de cirugías del equipo son } \\
5 \text { (rango; 4-6) cirugías } \\
\text { por semanas con las } \\
\text { restricciones sanitarias } \\
\text { (incluido cáncer de } \\
\text { tiroides) }\end{array}$ & 30.03.2020: 310 \\
\hline
\end{tabular}


Tabla 1. Cronograma general de cambios sanitarios críticos ministeriales, institucionales y Equipo de Cirugía de Cabeza y Cuello del Complejo Asistencial Barros Luco (CABL) desde el 03.03.2020 al 30.07.2020 (continuación)

Políticas ministeriales en relación a COVID-19

\section{Medidas instauradas en Medidas instauradas por \\ CABL}

Número de casos COVID-19 nuevos nacional por día*

03.04.2020: Circular C37 № 2 Racionalización de Uso de Equipos de Protección Personal (EPP) en el contexto de la atención de pacientes durante la pandemia COVID-19
08.04.2020:

08.04.2020: 430

Operacionalización

de insumos según recomendaciones EPP

Servicio Cirugía

16.04.2020: CVE 1752570 Resolución dispone uso

obligatorio de mascarillas en lugares públicos y privados

23.04.2020 ORD C21/ $\mathrm{N}^{0} 1218$ Orientaciones

complementarias para la gestión del recurso humano de la red asistencial pública, privada y FF. AA.

9.04.2020: Resolución exenta No 323 MINSAL

Reactivación de cirugías mayores ambulatorias o cuya hospitalización no exceda una noche

12.05.2020: CVE 1761196 Resolución dispone de medidas sanitarias que indica por brote de COVID-19 (aislamiento, cordones, aduanas sanitarias, medidas de protección, uso de mascarillas, fija precios de PCR SARS-CoV-2
23.04.2020: Optimización

de la gestión de recurso

humano ajustadas manteniendo laborales asistenciales y sistema de modalidad de rotación
12.05.2020:

Operacionalización de PCR SARS-CoV-2 preoperatoria en cirugías electivas por gestión clínica

13.05.2020: Reactivación de cirugías electivas no oncológicas por dirección
30.04.2020: Solicitud de PCR SARS-CoV-2 preoperatoria para pacientes electivos
13.05.2020: Addendum de consentimiento informado para cirugías electivas en Equipo $\mathrm{C}$ y $\mathrm{C}$ enviado a Unidad de Calidad para aprobación
14.05.2020: Nuevo

consentimiento

informado y encuesta

complementaria en

cirugías electivas por

Unidad de Calidad CABL
18.05.2020: Resolución exenta No 356 MINSAL

Instrucción de aumento de capacidad ventilatoria a los prestadores privados de salud cerrados de alta complejidad $(29 \%)$
18.05.2020: Tasa general de cirugías del equipo son 2,6 (rango: $1-5$ ) cirugías por semana con las restricciones sanitarias (incluido cáncer de tiroides)
13.05.2020: 2.660

14.05.2020: 2.656

\footnotetext{
*https://www.gob.cl/coronavirus/cifrasoficiales/\#resumen
} 


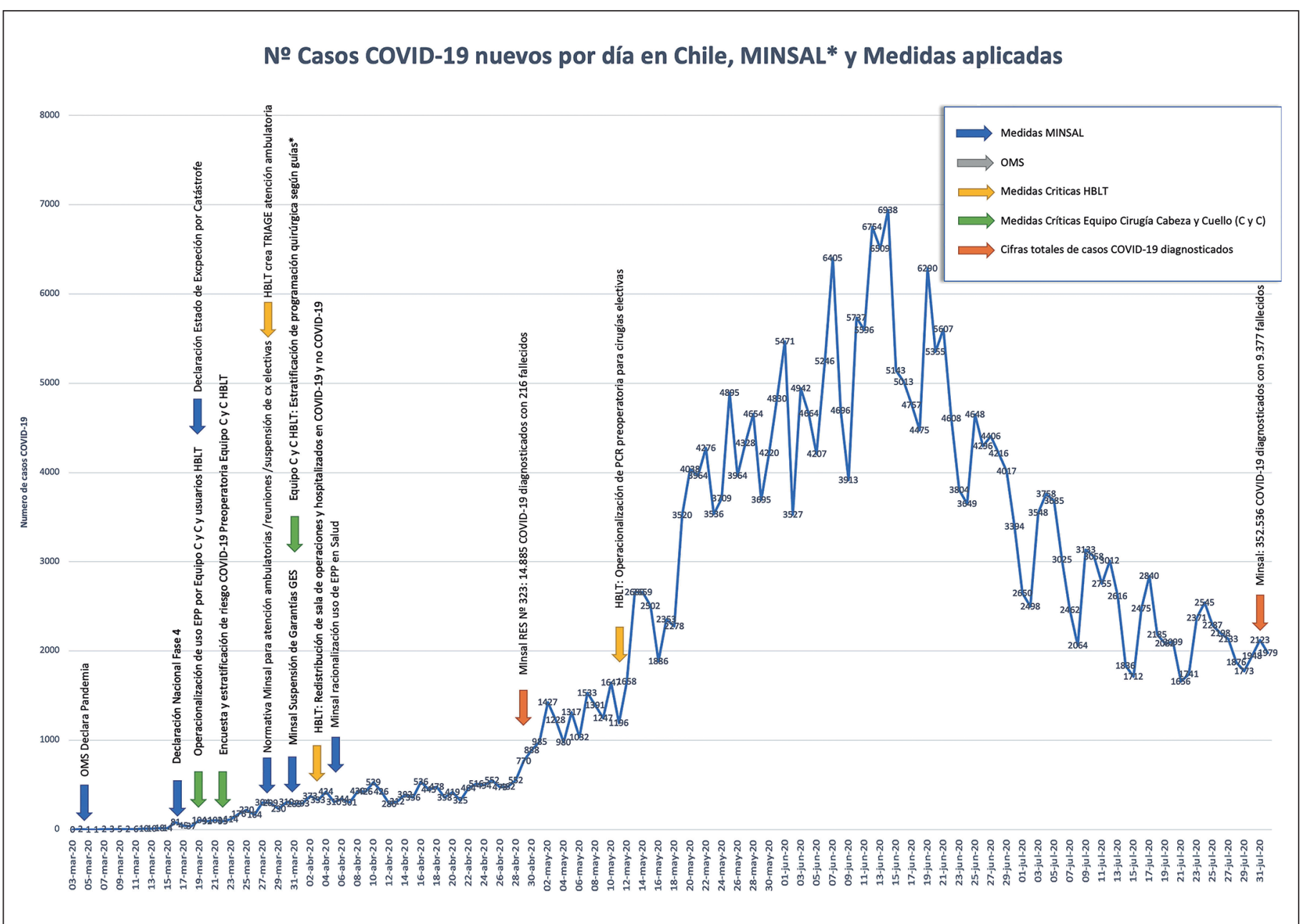

Figura 1. Número de casos COVID-19 nuevos por día en Chile y las medidas sanitarias ministeriales, institucionales Complejo Asistencial Barros Luco-Trudeau, y de Equipo de Cirugía de Cabeza y Cuello más relevantes desde el 03.03.2020 al 30.07.2020.

Tabla 2. Número de pacientes en atenciones ambulatorias y cirugías realizadas por Equipo de Cirugía de Cabeza y Cuello del Complejo Asistencial Barros Luco (CABL) durante los períodos del 3 de marzo al 30 de julio en 2019 y 2020, respectivamente

\begin{tabular}{|lcc|}
\hline & $\mathbf{2 0 1 9}(\mathbf{\%})$ & $\mathbf{2 0 2 0 *}(\mathbf{\%})$ \\
\hline $\begin{array}{l}\text { Atenciones ambulatorias totales Centro de Diagnóstico y Tratamiento (CDT) } \\
\text { Consultas nuevas en CDT }\end{array}$ & 1.079 & 374 \\
$\quad \begin{array}{l}\text { Consultas nuevas en CDT por cáncer } \\
\text { Consultas seguimiento clínico en CDT } \\
\text { Consultas seguimiento clínico en CDT por cáncer }\end{array}$ & $121(32,5)$ & $71(67,6)$ \\
255 \\
Intervenciones quirúrgicas realizadas en pabellón CABL & $204(28,9)$ & $147(57,6)$ \\
Intervenciones quirúrgicas CABL por cáncer & 144 & 61 \\
\hline
\end{tabular}

*Recomendaciones Plan de Contingencia COVID-10 Ord C27/N 808 en contexto de Fase 4 nacional por Subsecretaría de Redes Asistenciales Gobierno de Chile ${ }^{13}$. 
Tabla 3. Características generales de los pacientes operados durante Fase 4 de pandemia COVID-19 por Equipo de Cirugía Cabeza y Cuello Complejo Asistencial Barros Luco desde el 03.03.2020 al 30.07.2020

\begin{tabular}{|c|c|c|}
\hline Características & n de pacientes & $\%$ \\
\hline \multicolumn{3}{|l|}{ Género $(\mathrm{n}=61)$} \\
\hline Femenino & 40 & 65,5 \\
\hline Masculino & 21 & 34,4 \\
\hline Mediana edad al momento de la cirugía (mínimo-máximo) & $54,9 \quad(15-88)$ & \\
\hline Mediana días de hospitalización posoperatoria (mínimo-máximo) & $3,0 \quad(0-135)$ & \\
\hline \multicolumn{3}{|l|}{ Indicación de cirugía $(\mathrm{n}=61)$} \\
\hline Cáncer & 46 & 75,4 \\
\hline Refractariedad a tratamiento médico & 5 & 8,2 \\
\hline Malignidad incierta & 7 & 11,5 \\
\hline Urgencia & 3 & 4,9 \\
\hline \multicolumn{3}{|l|}{ Etapa $\operatorname{TNM}^{\alpha}(\mathrm{n}=42)$} \\
\hline Etapa I & 20 & 47,6 \\
\hline Etapa II & 16 & 38,1 \\
\hline Etapa III & 4 & 9,5 \\
\hline Etapa IV & 2 & 4,8 \\
\hline \multicolumn{3}{|l|}{ Infección por COVID-19 posoperatoria } \\
\hline Con infección* & 0 & 0 \\
\hline Sin infección & 61 & 100 \\
\hline \multicolumn{3}{|l|}{ Aplicación encuesta preoperatoria } \\
\hline Sí & 41 & 67,2 \\
\hline No & 20 & 32,8 \\
\hline \multicolumn{3}{|l|}{ Toma de PCR SARS-CoV-2 48 h precirugía } \\
\hline Sí & 12 & 19,7 \\
\hline No & 49 & 80,3 \\
\hline \multicolumn{3}{|l|}{ Fallecidos } \\
\hline Por otras causas & 3 & 4,9 \\
\hline Por COVID-19 & 0 & 0 \\
\hline
\end{tabular}

${ }^{*} \mathrm{CDC}$ considera infección nosocomial dentro de los 14 días posexposisción ${ }^{16} .{ }^{\alpha} \mathrm{TNM}$ de los usuarios con primario de cabeza y cuello.

Con respecto a los números absolutos de atenciones ambulatorias por el ECCC del CABL durante los períodos del 3 de marzo al 30 de julio en 2019 y 2020, respectivamente, son representados en la Tabla 2. Las atenciones ambulatorias de 2019 a 2020 se redujeron en un $63 \%$, con mayor número de atenciones ambulatorias en pacientes oncológicos nuevos y seguimientos clínicos oncológicos.

\section{Discusión}

La actual pandemia ha impactado drásticamente la actividad de todos los centros quirúrgicos del mundo, y nuestro departamento no estuvo exento de este efecto deletéreo. Un esfuerzo estratégico local permitió implementar una serie de medidas protocolizadas en el área preoperatoria, operatoria y ambulatoria según la evidencia disponible y consensos de expertos en el área, con la finalidad de mitigar el efecto de esta pandemia COVID-19 en nuestros usuarios y preservar la atención en los casos no diferibles. Esto adosado a los simultáneos cambios estratégicos ministeriales e institucionales que se centraron en la atención de los pacientes $\mathrm{CO}$ VID-19, con la redistribución de equipos y recursos humanos. Secundario a estas mayores limitaciones clínicas, la reducción progresiva de la atención ambulatoria con casos nuevos y la postergación de las cirugías electivas en todos los servicios de cirugía; 
la práctica clínica de cirugía de cabeza y cuello se vio drásticamente disminuida durante el período inicial de casos COVID-19.

Sumado a los reportes de morbimortalidad y a la palpable limitación de los recursos, las sociedades quirúrgicas internacionales afectadas inicialmente publicaron simultáneamente sistemas de estratificación de las intervenciones, posponiendo todo aquel procedimiento quirúrgico que pudiera ser diferido durante la pandemia COVID-19, en consideración a los riesgos descritos en la literatura ${ }^{26-29}$. Esto indujo una reducción drástica de la capacidad quirúrgica a escala mundial, llegando en algunos lugares al $90 \%$ de reducción ${ }^{30}$. Una reciente publicación evaluó el impacto en 71 países con el uso del modelo Bayesiano de regresión durante el período de 12 semanas (aproximadamente 360 días) de iniciada la pandemia COVID-19, demostró una tasa estimada de cancelación de cirugías no urgentes de 23\%-77\% para cirugías oncológicas, $71 \%-87 \%$ para cirugías no oncológicas y un 17\%-38\% para cirugías obstétricas $^{31}$. En nuestro estudio de los primeros 150 días de pandemia, se generó una reducción del $58 \%$ en la capacidad quirúrgica. Según datos del Minsal, en nuestro país hubo una disminución al $52 \%$ en la actividad quirúrgica en el período comprendido entre enero y septiembre de 2020, en comparación con el año 2019.

Otra medida aplicada en ECCC del CABL fue la realización de ERECoVp en 41 pacientes programados. Un protocolo de tamizaje preoperatorio similar reportado y utilizado en pacientes con patología de cabeza y cuello, corresponde al Protocolo de Milán recientemente publicado ${ }^{32}$. Este protocolo realizaba una entrevista telefónica para detectar la presencia de síntomas relacionados con COVID-19 en 23 pacientes, luego de lo cual ingresaban como asintomáticos para continuar con su estudio bioquímico general y TC de tórax un día previo a la cirugía, $\mathrm{y}$ en caso de que todo lo anterior se encontraran negativos se procedía con la cirugía programada ${ }^{32}$. Mehanna et al., publicó en junio, un consenso internacional de 35 sociedades de cirugía de cabeza y cuello que forman parte del Head and Neck Cancer International Group (HNCIG), haciendo énfasis en el reconocimiento del COVID-19 preoperatoriamente ${ }^{13}$. Hasta la fecha no existen protocolos estandarizados del manejo preoperatorio de pacientes en cabeza y cuello durante el período COVID-19, por lo tanto, siguen siendo recomendaciones acordes con la evidencia actualmente disponible ${ }^{33}$.

La aplicación de pruebas SARS-CoV-2 preoperatorias para pacientes ha sido uno de los tantos pilares de tamizaje en pacientes que serán sometidos a cirugía programada con o sin cuarentena preoperatoria, y nuestro establecimiento no estuvo ajeno a la aplicación de esta herramienta de detección, alcanzando el $20 \%$ en nuestra cohorte. La detección de la subpoblación asintomática o prodrómica en el contexto preoperatorio toma relevancia debido a la alta tasa de morbimortalidad en los pacientes oncológicos y electivos operados con co-infección SARS-CoV-2, además de considerar que los individuos asintomáticos poseen una importante contribución a la transmisibilidad de la enfermedad con una carga viral similar a sus contrapartes sintomáticas, la que pudiera no solo darse en el ambiente comunitario, sino también hospitalario al no ser detectados dirigidamente ${ }^{34-37}$. Es por ello que hasta la fecha algunos consensos de expertos recomiendan el estudio de PCR SARS-CoV-2 preoperatoria 24 a 72 horas previo a la cirugía programada de cabeza y cuello ${ }^{10,32}$. Es fundamental mencionar que la PCR SARS-CoV-2 preoperatoria posee una tasa de falsos negativos, y el compromiso de la vía aerodigestiva por SARS-CoV-2 es considerado de alto riesgo de aerosolización y contagio por COVID-19, por consiguiente, el uso correcto y periódico de los EPP es esencial para mitigar el riesgo de transmisión ${ }^{25,38,39}$.

Las limitaciones de este estudio son acordes con las limitaciones de todo estudio de naturaleza retrospectiva. A pesar de ser un reporte unicéntrico nacional, pudiera ser de utilidad para proyectar el efecto de la era COVID-19 en otros centros de salud nacional, y evaluar la replicación de estas medidas sanitarias locales empleadas, considerando que $\mathrm{CABL}$ abarca una extensa población asignada cercana al millón doscientas mil personas con un total de 739 camas totales para usuarios adultos ${ }^{40}$. Otra limitación del estudio es que 20 pacientes no fueron testeados con PCR SARS-CoV-2/ERECoVp, debido a que sus intervenciones fueron realizadas previo a las restricciones del Plan de Contingencia MINSAL COVID-19 y las limitaciones de la evidencia científica disponible. Sin embargo, ninguno de estos pacientes presentó sintomatología atribuible a infección por COVID-19 en los 14 días posterior al alta hospitalaria luego del seguimiento clínico activo por parte del equipo.

\section{Conclusión}

La pandemia por COVID-19 generó un drástico cambio en la ejecución del cuidado del usuario de cirugía de cabeza y cuello a nivel nacional y mundial. Esta pandemia COVID-19 motivó que países y centros especializados de cirugía de cabeza y 
cuello creáramos guías de manejo y priorización de tratamiento con protocolo de manejo activo preoperatorio para pacientes durante este período. Consideramos que la importancia de nuestra publicación es reportar los resultados de una serie de pacientes sometidos a cirugía de cabeza y cuello en el período inicial de la pandemia, y exponer que, tomando las medidas apropiadas para el flujo de pacientes tanto en la atención abierta como cerrada, es posible brindar un manejo apropiado minimizando los riesgos inherentes en los pacientes más críticos durante el período COVID-19. Los flujogramas de acción en cirugía de cabeza y cuello en el contexto de la pandemia por COVID-19 continuarán en constante evolución a lo largo del tiempo según la evidencia científica disponible y el desarrollo de esta pande- mia, por ello, esfuerzos colaborativos nacionales de la especialidad son esenciales para evaluar en conjunto el efecto COVID-19 en los pacientes de cirugía de cabeza y cuello.

\section{Responsabilidades éticas}

Protección de personas y animales. Los autores declaran que para esta investigación no se han realizado experimentos en seres humanos ni en animales.

Confidencialidad de los datos. Los autores declaran que en este artículo no aparecen datos de pacientes.

Conflictos de interés: no hay.

\section{Bibliografía}

1. Mouton C, Hirschmann MT, Ollivier M, Seil R, Menetrey J. COVID-19 - ESSKA guidelines and recommendations for resuming elective surgery. J Exp Orthop. 2020; 7:28. DOI: 10.1186/s40634-02000248-4.

2. Díaz A, Sarac BA, Schoenbrunner AR, Janis JE, Pawlik TM. Elective surgery in the time of COVID-19. Am J Surg. 2020;219:900-2. DOI: $10.1016 / j$. amjsurg.2020.04.014.

3. Lei S, Jiang F, Su W, Chen C, Chen J, Mei W, et al. Clinical characteristics and outcomes of patients undergoing surgeries during the incubation period of COVID-19 infection. EClinicalMedicine. 2020;21:100331. DOI: 10.1016/j. eclinm.2020.100331.

4. COVIDSurg Collaborative. Mortality and pulmonary complications in patients undergoing surgery with perioperative SARS-CoV-2 infection: an international cohort study. Lancet. 2020;396(10243):27-38. DOI: 10.1016/ S0140-6736(20)31182-X.

5. Doglietto F, Vezzoli M, Gheza F, Lussardi GL, Domenicucci M, Vecchiarelli L, et al. Factors Associated With Surgical Mortality and Complications Among Patients With and Without Coronavirus Disease 2019 (COVID-19) in Italy. JAMA 2020;155:691-702. DOI: 10.1001/ jamasurg.2020.2713.

6. Aminian A, Safari S, Razeghian-Jahromi A, Ghorbani M, Delaney CP. COVID-19
Outbreak and Surgical Practice: Unexpected Fatality in Perioperative Period. Ann Surg. 2020;272:e27-e9. DOI: $10.1097 /$ SLA.0000000000003925.

7. $\mathrm{Wu} \mathrm{V}$, Noel CW, Forner D, Zhang ZJ, Higgins KM, Enepekides DJ, et al. Considerations for head and neck oncology practices during the coronavirus disease 2019 (COVID-19) pandemic: Wuhan and Toronto experience. Head Neck. 2020;42:1202-8. DOI: 10.1002/ hed.26205.

8. Riva G, Pizzo C, Fassone E, Pecorari G. Head and neck cancer surgery in COVID-19 pandemic in Northern Italy. Oral Oncol 2020;107:104835. DOI: $10.1016 / \mathrm{j}$. oraloncology.2020.104835.

9. Tzeng CD, Teshome M, Katz MHG, Weinberg JS, Lai SY, Antonoff MB, et al. Cancer Surgery Scheduling During and After the COVID-19 First Wave: The MD Anderson Cancer Center Experience. Ann Surg. 2020;272:e106-e11.DOI: 10.1097/ SLA.0000000000004092.

10. MD Anderson Head and Neck Surgery Treatment Guidelines Consortium; Consortium members, Maniakas A, Jozaghi Y, Zafereo ME, Sturgis EM, Su SY, Gillenwater AM, et al. Head and neck surgical oncology in the time of a pandemic: Subsite-specific triage guidelines during the COVID-19 pandemic. Head Neck 2020;42:1194-201. DOI: 10.1002/hed.26206.

11. Ota I, Asada Y. The impact of preoperative screening system on head and neck cancer surgery during the COVID-19 pandemic: Recommendations from the nationwide survey in Japan. Auris Nasus Larynx 2020;47:687-91. DOI: 10.1016/j. anl.2020.05.006.

12. Moletta L, Pierobon ES, Capovilla G, Costantini M, Salvador R, Merigliano $\mathrm{S}$, et al. International guidelines and recommendations for surgery during Covid-19 pandemic: A Systematic Review. Int J Surg. 2020;79:180-8. DOI: $10.1016 /$ j.ijsu.2020.05.061.

13. Mehanna H, Hardman JC, Shenson JA, Abou-Foul AK, Topf MC, AlFalasi M, et al. Recommendations for head and neck surgical oncology practice in a setting of acute severe resource constraint during the COVID-19 pandemic: an international consensus. Lancet Oncol. 2020;21:e350-e9. DOI: 10.1016/S14702045(20)30334-X.

14. Silva-Figueroa A, Gallego-Cifuentes A, Veloso-Olivares M. Recomendaciones generales en cirugía oncológica de cabeza y cuello durante la pandemia COVID-19. Revista de Cirugía. 2020;72:361-8. DOI: $10.35687 / \mathrm{s} 2452-45492020004820$.

15. Paleri V, Hardman J, Tikka T, Bradley P, Pracy P, Kerawala C. Rapid implementation of an evidence-based remote triaging system for assessment of suspected referrals and patients with head and neck cancer on follow-up after treatment during the COVID-19 pandemic: Model for international collaboration. Head Neck 2020;42:167480. DOI: $10.1002 /$ hed.26219. 
16. Baud G, Brunaud L, Lifante JC, Tresallet C, Sebag F, Bizard JP, et al. Endocrine surgery during and after the COVID-19 epidemic: Expert guidelines from AFCE. J Visc Surg. 2020;157(3S1):S43-S9. DOI: 10.1016/j. jviscsurg.2020.04.018.

17. Aygun N, Iscan Y, Ozdemir M, Soylu S, Aydin OU, Sormaz IC, et al. Endocrine Surgery during the COVID-19 Pandemic: Recommendations from the Turkish Association of Endocrine Surgery. Sisli Etfal Hastan Tip Bul. 2020;54:117-31. DOI: 10.14744/SEMB.2020.65902.

18. Jozaghi $Y$, Zafereo ME, Perrier ND, Wang JR, Grubbs E, Gross ND, et al. Endocrine surgery in the Coronavirus disease 2019 pandemic: Surgical Triage Guidelines. Head Neck. 2020;42:1325-8. DOI: 10.1002/hed.26169.

19. Subsecretario de redes asistenciales, Recomendaciones Plan de Contingencia COVID-10 Ord C27/ $\mathrm{N}^{\circ} 808$ en contexto de Fase 4 nacional (2020). Subsecretaría de Redes Asistenciales. [Available from: https://www.minsal.cl/nuevo-coronavirus2019-ncov/informe-tecnico/].

20. Mañalich J. Suspende Garantías de Oportunidad de las Garantías Explícitas en Salud en los problemas de salud que Indica. 2020. Ministerio de Salud de Chile. [Available from: https://www. minsal.cl/nuevo-coronavirus-2019-ncov/ informe-tecnico/]

21. Mañalich J, Piñera S. Alerta Sanitaria por el período que se señala y otorga facultades extraordinarias que indica por Emergencia de Salud Pública de Importancia Internacional (ESPII) por brote del nuevo coronavirus (2019NCOV). 2020. [Available from: https:// www.minsal.cl/nuevo-coronavirus-2019ncov/informe-tecnico/].

22. Subsecretario de Redes Asistenciales. Protocolo de uso de equipos de protección personal en la prevención de transmisión de COVID-19 - SARS-CoV-2 2020. [Available from: https://www.minsal.cl/ nuevo-coronavirus-2019-ncov/informetecnico/].

23. Subsecretario de Redes Asistenciales. Ord. C37 N 670 Herramientas para evaluar estado de implementación de medidas de prevención de IAAS asociadas al virus SARS-CoV-2. 2020. [Available from: https://www.minsal.cl/nuevo-coronavirus- 2019-ncov/informe-tecnico/].

24. Lauer SA, Grantz KH, Bi Q, Jones FK, Zheng Q, Meredith HR, et al. The Incubation Period of Coronavirus Disease 2019 (COVID-19) From Publicly Reported Confirmed Cases: Estimation and Application. Ann Intern Med. 2020;172:577-82. DOI: 10.7326/M200504 .

25. Givi B, Schiff BA, Chinn SB, Clayburgh D, Iyer NG, Jalisi S, et al. Safety Recommendations for Evaluation and Surgery of the Head and Neck During the COVID-19 Pandemic. JAMA Otolaryngol Head Neck Surg. 2020. DOI: 10.1001/ jamaoto.2020.0780.

26. Coimbra R, Edwards S, Kurihara H, Bass GA, Balogh ZJ, Tilsed J, et al. European Society of Trauma and Emergency Surgery (ESTES) recommendations for trauma and emergency surgery preparation during times of COVID-19 infection. Eur J Trauma Emerg Surg. 2020;46:505-10. DOI: 10.1007/s00068-020-01364-7.

27. Balibrea JM, Badia JM, Rubio Pérez I, Martin Antona E, Álvarez Pena E, García Botella S, et al. Surgical Management of Patients With COVID-19 Infection. Recommendations of the Spanish Association of Surgeons. Cir Esp. 2020;98(5):251-9. DOI: 10.1016/j. ciresp.2020.03.001.

28. American College of Surgeons. COVID-19: Series Title: Information for Surgeons. Guidance for Triage of NonEmergent Surgical Procedures. 2020. [Available from: https://www.facs.org/ covid-19/clinical-guidance/triage.

29. Royal College of Surgeons. Updated Series Title: Guidance for Surgeons working during the pandemic, Intercollegiate General Surgery Guidance on COVID-19. 2020. [Available from: https://www.rcseng.ac.uk/coronavirus/ joint-guidance-for-surgeons-v2/].

30. Argenziano M, Fischkoff K, Smith CR. Surgery Scheduling in a Crisis. N Engl J Med. 2020;382:e87. DOI: 10.1056/ NEJMc2017424.

31. Soltany A, Hamouda M, Ghzawi A, Sharaqi A, Negida A, Soliman S, et al. A scoping review of the impact of COVID-19 pandemic on surgical practice. Ann Med Surg (Lond). 2020;57:24-36. DOI: 10.1016/j.amsu.2020.07.003.

32. Ferrari M, Paderno A, Giannini L,
Cazzador D, Ciardiello C, Carretta G, et al. COVID-19 screening protocols for preoperative assessment of head and neck cancer patients candidate for elective surgery in the midst of the pandemic: A narrative review with comparison between two Italian institutions. Oral Oncol. 2021;112. DOI: 10.1016/j. oraloncology.2020.105043.

33. Brindle ME, Gawande A. Managing COVID-19 in Surgical Systems. Ann Surg. 2020;272:e1-e2. DOI: 10.1097/ SLA.0000000000003923.

34. Mizumoto K, Kagaya K, Zarebski A, Chowell G. Estimating the asymptomatic proportion of coronavirus disease 2019 (COVID-19) cases on board the Diamond Princess cruise ship, Yokohama, Japan, 2020. Euro Surveill. 2020;25(10). DOI: 10.2807/1560-7917. ES.2020.25.10.2000180.

35. Zou L, Ruan F, Huang M, Liang L, Huang $\mathrm{H}$, Hong Z, et al. SARS-CoV-2 Viral Load in Upper Respiratory Specimens of Infected Patients. N Engl J Med. 2020;382:1177-9. DOI: 10.1056/ NEJMc2001737.

36. Hu Z SC, Xu C, Jin G, Chen Y, $\mathrm{Xu} \mathrm{X}, \mathrm{Ma} \mathrm{H}$, et al. Clinical characteristics of 24 asymptomatic infections with COVID-19 screened among close contacts in Nanjing, China. Sci China Life Sci. 2020;63:706-11. DOI: 10.1007/s11427020-1661-4.

37. Aguilar JB FJ, Westafer LM, Gutiérrez JB. Investigating the Impact of Asymptomatic Carriers on COVID-19 Transmission. COVID-19 Publications by UMMS Authors. 2020. DOI: 10.1101/2020.03.18.20037994.

38. Surkova E, Nikolayevskyy V, Drobniewski F. False-positive COVID-19 results: hidden problems and costs. Lancet Respir Med. 2020;8:1167-8. DOI: 10.1016/S22132600(20)30453-7.

39. Yuen E, Fote G, Horwich P, Nguyen SA, Patel R, Davies J, et al. Head and neck cancer care in the COVID-19 pandemic: A brief update. Oral Oncol. 2020;105:104738. DOI: 10.1016/j. oraloncology.2020.104738.

40. Dirección Hospital Barros Luco Trudeau. Cuenta Pública. 2020. [Available from: http://www.hospitalbarrosluco.cl/ institucion/cuentas-publicas/]. 